Forum 2018 $33: 72$

https://doi.org/10.1007/s12312-018-0404-5

Online publiziert: 27. Februar 2018

๑) Springer Medizin Verlag GmbH, ein Teil von Springer Nature 2018

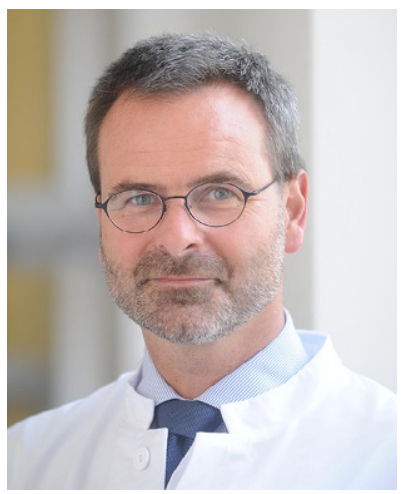

Die gesundheitspolitischen Diskussionen der letzten Wochen waren geprägt von der Auseinandersetzung um die Zweiklassen-Medizin, in der Privatversicherte angeblich besser behandelt würden als die Mitglieder der GKV. In einem Interview im Deutschlandfunk sagte SPD-Gesundheitsexperte Karlheinz Lauterbach im Februar: „Wir wissen von Zentren für Krebsbehandlung, dass diese bessere Ergebnisse haben als keine spezialisierten Kliniken, und wir wissen, dass der Anteil der Privatpatienten in diesen Zentren viel, viel höher ist.“ Weil Privatpatienten einen besseren Zugang zu den wenigen Spezialisten hätten, würden sie besser behandelt.

Auf die Frage nach den Belegen für diese Behauptung musste Lauterbach allerdings passen. (Auch wenn beide Behauptungen richtig wären, dann ist damit noch nicht gesichert, dass die logische Verknüpfung stimmt.) Kein Wunder, denn über die Diagnostik und Therapie eines Krebspatienten in Deutschland entscheidet nicht der Versicherungsstatus und auch nicht der limitierte Zugang zu herausragenden Spezialisten. Wichtig ist vielmehr ein solides Qualitätsmanagement, das die Behandlung multidiszipli-

\title{
„Über die Therapieergebnisse eines Krebspatienten in Deutschland entscheidet nicht der Versicherungsstatus."
}

när organisiert, auf erfahrene Teams baut und die leitliniengerechte Behandlung einbezieht. Dass sich ein solches Qualitätsmanagement in der Tat in die Fläche bringen lässt, davon zeugt das Zentrenmodell der Deutschen Krebsgesellschaft. Mehr als 1000 zertifizierte Zentren unterziehen sich regelmäßigen Audits das zahlt sich aus. Auf dem Deutschen Krebskongress wurde eine Untersuchung an mehr als 25.000 Prostatakrebspatienten vorgestellt, die nach einer radikalen Prostatektomie eine Anschlussheilbehandlung absolviert hatten. Diejenigen, die an einem DKG-zertifizierten Zentrum operiert worden waren, schnitten besser ab als die aus nichtzertifizierten Zentren. Weitergehende Analysen müssen noch das Ergebnis bestätigen, aber in Verbindung mit Analysen aus anderen Tumorentitäten verdichtet sich aktuell die Datenlage zugunsten einer Zentrumsbehandlung von Krebspatienten.

Wie steht es um die gesundheitspolitische Zukunft in Deutschland? Im Entwurf zum Koalitionsvertrag vom 7. Februar sind die Herausforderungen skizziert: Pflegenotstand, Sektorengrenzen, die sich wenig am Bedarf der Patienten orientieren, und ländliche Gebiete mit Versorgungslücken. Manche Forderungen klingen vertraut: Medizinische Innovationen sollen schneller in die Regelversorgung gelangen, dafür wird eine Beschleunigung der Verfahren in Gemeinsamen Bundesausschuss angestrebt. Der Innovationsfonds soll über das Jahr 2019 hinaus weiterbestehen (mit einer Reduktion auf 200 Millionen Euro jährlich), die Vernetzung von Strukturen verbessert und die Patientenorientierung vorangetrieben werden. (Einen Einblick in dieses komplexe Thema bieten die Beiträge in diesem Heft.)
Vieles davon haben medizinische Fachgesellschaften und Experten in der vergangenen Legislaturperiode gefordert oder angedacht. Einerseits freut es mich, wenn diese Ziele aufgegriffen werden, andererseits bin ich skeptisch. Zum Beispiel beim Pflegenotstand: Zwar hat man sich verständigt, Personaluntergrenzen für alle bettenführenden Abteilungen festlegen zu wollen. Die Beschränkung auf sogenannte pflegesensitive Abteilungen scheint damit vom Tisch. Bis wann und wie genau die vage angekündigten Maßnahmen vor dem Hintergrund des Fachkräftemangels konkretisiert werden, ist allerdings offen. Und wie das Ansehen der Pflegeberufe durch Weiterqualifikation, Übernahme verantwortlicher Tätigkeiten z.B. in der fachonkologischen Pflege zusätzlich zu einer besseren Bezahlung gestärkt werden könnte, wird im Koalitionsvertrag ebenfalls nicht thematisiert. Oder die patientenorientierte Palliativversorgung: Palliativmediziner werden oft zu spät hinzugezogen, wenn für die Betroffenen nur noch wenig Zeit bleibt oder ihnen die Möglichkeit fehlt, ihre Therapiewünsche zu artikulieren. Die Folge ist oft eine überengagierte Versorgung Krebskranker am Lebensende. Bei der Suche nach den richtigen strukturellen Maßnahmen für diese Probleme helfen intelligente Forschung, Untersuchungen, Belege, keine Behauptungen oder parteipolitisches Kalkül.

Ihr

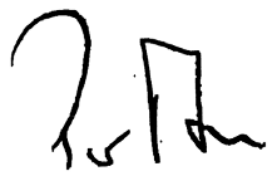

Peter Albers

Präsident der Deutschen Krebsgesellschaft 